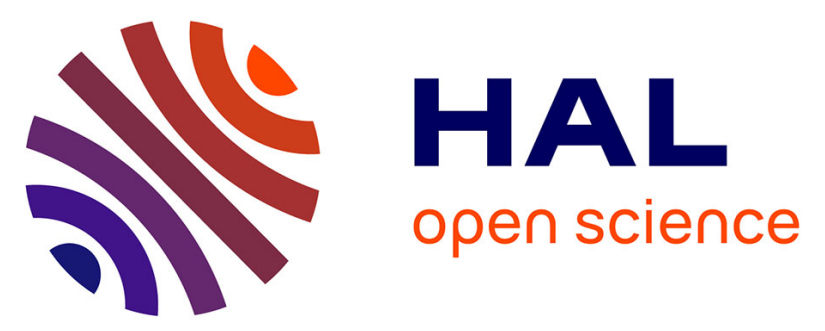

\title{
HD-RTI: an adaptive multi-light imaging approach for the quality assessment of manufactured surfaces
} Marvin Nurit, Gaëtan Le Goïc, David Lewis, Yuly Castro, Abir Zendagui, Hermine Chatoux, Hugues Favrelière, Stéphane Maniglier, Pierre Jochum, Alamin Mansouri

\section{To cite this version:}

Marvin Nurit, Gaëtan Le Goïc, David Lewis, Yuly Castro, Abir Zendagui, et al.. HD-RTI: an adaptive multi-light imaging approach for the quality assessment of manufactured surfaces. Computers in Industry, 2021, 132, pp.103500. 10.1016/j.compind.2021.103500 . hal-03369237

\section{HAL Id: hal-03369237 https://hal.science/hal-03369237}

Submitted on 7 Oct 2021

HAL is a multi-disciplinary open access archive for the deposit and dissemination of scientific research documents, whether they are published or not. The documents may come from teaching and research institutions in France or abroad, or from public or private research centers.
L'archive ouverte pluridisciplinaire $\mathbf{H A L}$, est destinée au dépôt et à la diffusion de documents scientifiques de niveau recherche, publiés ou non, émanant des établissements d'enseignement et de recherche français ou étrangers, des laboratoires publics ou privés. 


\title{
HD-RTI: an adaptive multi-light imaging approach for the quality assessment of manufactured surfaces
}

\author{
Marvin Nurit ${ }^{\mathrm{a}}$, Gaëtan Le Goïc ${ }^{\mathrm{a}}$, David Lewis ${ }^{\mathrm{a}}$, Yuly Castro ${ }^{\mathrm{a}}$, Abir \\ Zendagui $^{\mathrm{a}}$, Hermine Chatoux ${ }^{\mathrm{a}}$, Hugues Favrelière ${ }^{\mathrm{b}}$, Stéphane Maniglier ${ }^{\mathrm{c}}$, \\ Pierre Jochum ${ }^{\mathrm{d}}$, Alamin Mansouri ${ }^{\mathrm{a}}$ \\ ${ }^{a}$ ImViA laboratory - EA 7534, Université de Bourgogne, Dijon, France \\ ${ }^{b}$ Symme laboratory -EA 4144, Université Savoie Mont-Blanc, Annecy, France \\ ${ }^{c}$ Technical Centre for Mechanical Industry (CETIM), Cluses, France \\ ${ }^{d}$ Francéclat, Technical department, Besançon, France
}

\begin{abstract}
Reflectance Transformation Imaging (RTI) is a technique for estimating surface local angular reflectance from a set of stereo-photometric images captured with variable lighting directions. The digitization of this information fully fits into the industry 4.0 approach and makes it possible to characterize the visual properties of a surface. The proposed method, namely HD-RTI, is based on the coupling of RTI and HDR imaging techniques. This coupling is carried out adaptively according to the response at each angle of illumination. The proposed method is applied to five industrial samples which have high local variations of reflectivity because of their heterogeneity of geometric texture and/or material. Results show that coupling HDR and RTI improves the relighting quality compared to RTI, and makes the proposed approach particularly relevant for glossy and heterogeneous surfaces. Moreover, HD-RTI enhances significantly the characterization of the local angular reflectance, which leads to more discriminating visual saliency maps, and more generally to an increase in robustness for visual quality assessment tasks.
\end{abstract}

Keywords: Material Appearance, Machine vision, Quality inspection

\section{Introduction}

Surfaces are the location of complex and multi-physical interactions. Particularly, the interaction with its luminous environment is currently the subject of several researches in various application fields such as cultural heritage [1, 2], 5 bio-medical, forensic [3] and the manufacturing industry for the purpose of automation of inspection tasks [4, 5], or for failure analysis [6]. In the latter field, two objectives are pursued through this kind of analysis. The first objective is

Email address: Marvin_Nurit@etu.u-bourgogne.fr (Marvin Nurit) 


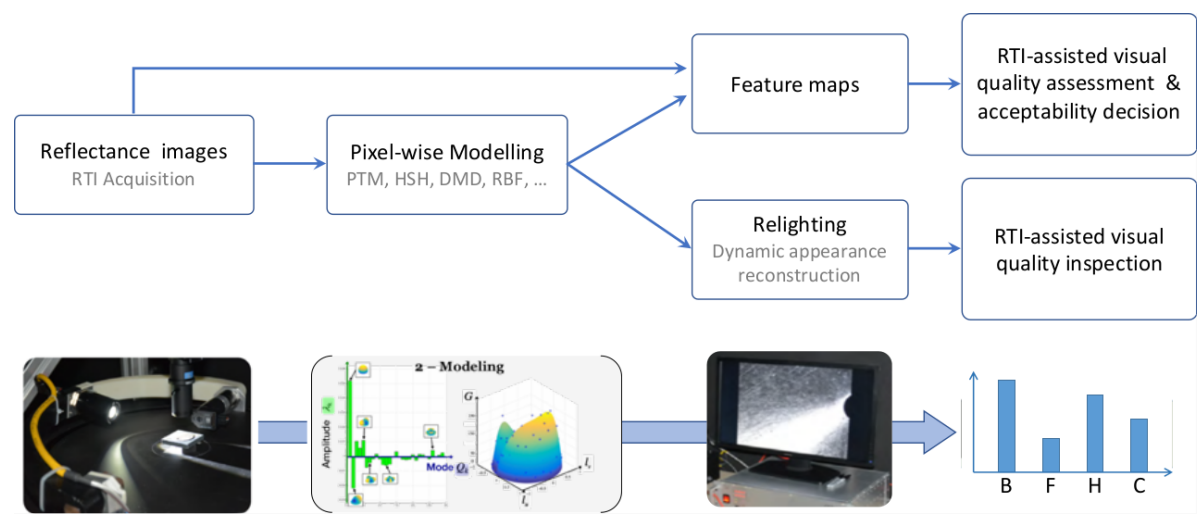

Figure 1: RTI-based flowchart for visual quality assessment of manufactured surfaces in industry

a better understanding of how the surface was fabricated in order to better control the process and manufacturing aspects 7,8 . The second relies on better understanding and mastering of how surfaces are perceived, which has become a potent lever of value creation for many industries [9].

The interaction between a surface and its luminous environment can be assessed through different approaches. The most exhaustive technique is the measurement of the Bidirectional Reflectance Distribution Function (BRDF)

15 [10. However, this technique requires heavy setups and is very time consuming, making it inadequate when it comes to many industrial applications. Simplified approaches have thus been developed based on the selection and measurement of the most relevant quantities regarding the surface properties and/or functionalities to be mastered. In this paper, we focus on Reflectance Transformation 20 Imaging (RTI), originally developed as Polynomial Texture Mapping [11, 12]. In the industrial context, RTI imagery data can either be used to extract features for decision making on conformity or used as a medium for visual assessment through relighting. Figure 1 presents such an exploitation pipeline of RTI data in the industry.

25 The following section will briefly present the RTI technique and highlight one of its limits: a dynamic range that is too low when acquiring highly specular surfaces. The proposed method (augmenting RTI images with the HDR imaging technique) is presented in section 3 . The section 4 presents our experimental protocol and results on three surface samples with distinct reflectance properties 30 in terms of isotropy and dynamic range. As an application case, we present a fourth surface to compare its saliency map computed from low dynamic and high dynamic acquisition (Section 5). Finally, we conclude with the gain of the proposed method. 


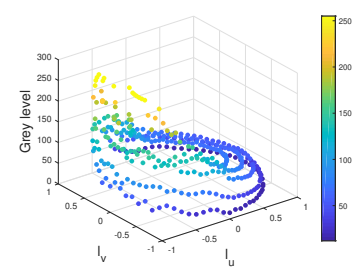

(a)

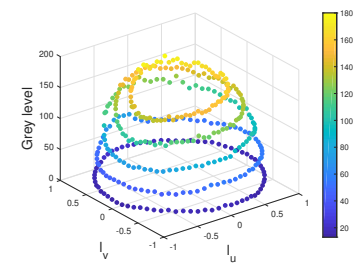

(b)

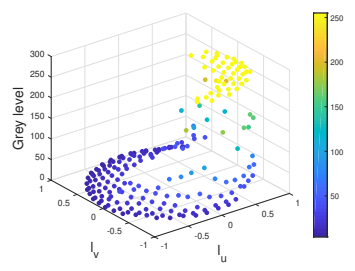

(c)

Figure 2: Example of RTI discrete luminance point clouds for three different pixels.

\section{Reflectance Transformation Imaging}

An RTI acquisition consists in acquiring a set of images of an observed surface, each image corresponding to a specific direction of illumination. The camera stands at a fixed position, generally orthogonal to the inspected surface. As a result of the acquisition process we obtain a stack of stereo-photometric images. On this set of images, each pixel can be considered as a discrete measure

40 of the luminance of the corresponding point in the observed scene. Figure 2 presents three different pixels' responses at each illumination angle. The gray level of the pixels varies for each direction of illumination. Its value is either within the acquisition range, underexposed (dark blue points) or overexposed (yellow points).

45 From stereophotometric images, the next step is the modelling whose aim is to build a continuous representation from discrete acquired data. The continuous model is necessary for the operation named relighting, which consists in virtually varying the light direction [13. Relighting is used generally for visual assessment but can also be used for further processing such as material appear50 ance characterization [14, 15]. This can be very helpful for human operators in charge of control/inspection since it can help avoid handling of the objects and enables automation of the task.

To build a continuous model, it's common to use data fitting by approximation [16] or interpolation [17]. Therefore, It is essential to have an efficient

55 approximation model allowing for the purposes of relighting. In this context, recent works on the Discrete Modal Decomposition have shown good performance [18 in comparison to the Polynomial Texture Mappings (PTM) 19] and the Hemi-Spherical Harmonics (HSH) 20 approaches. Interpolation-based approaches such the one based on Radial Basis Functions (RBFs) belong to a different category since the modelling is local, but whose performance is still good especially for relighting purposes [21, 22].

Moreover, some geometric 2] and photometric indicators [23] can be derived from this information in order to better understand the link of local topography on visual appearance. These indicators answer to different application needs

65 24, 25, as illustrated in the flowchart presented in Figure1. An RTI application in industry consists, for instance, in highlighting the salient pixels/areas in order to facilitate the quality assessment [26] (see Section 5). 


\subsection{Pixel-level and image-level dynamic limitation}

The camera in an RTI setup is used as a photosensor capturing and sampling 70 the surface luminance at each point of the scene. One important characteristic of a camera is its dynamic range, which is actually the ratio between the largest and the smallest signal values. Recent sensor's dynamic range is about $1 / 10^{5}$ (approximately $60 \mathrm{~dB}$ ). In comparison, human vision sensitivity is around $1 / 10^{9}$ $(90 \mathrm{~dB})$. In the case of a conventional RTI acquisition, this dynamic limitation is

75 particularly detrimental. Indeed, the light amount reflected by a surface varies greatly with the illumination angle (incidence and azimuth).

Figure 3 presents images obtained for two surfaces (paper and metal materials) at three different illumination angles (illustrated by the spherical representation at the bottom right corner of each image).

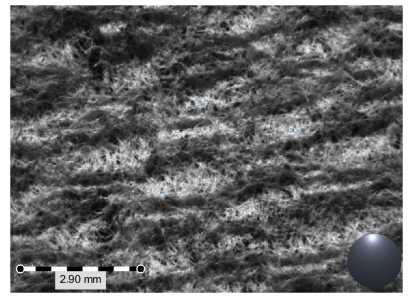

(a) Paper: $r_{S}=0 \%$

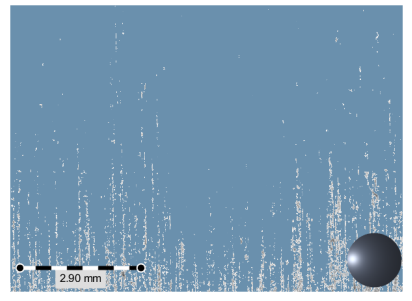

(d) Metal: $r_{S}=94 \%$

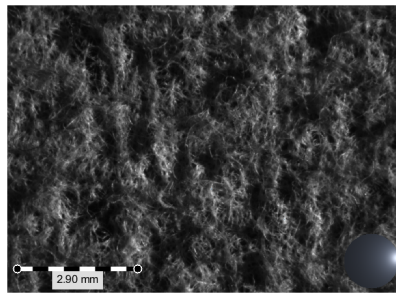

(b) Paper: $r_{S}=0 \%$

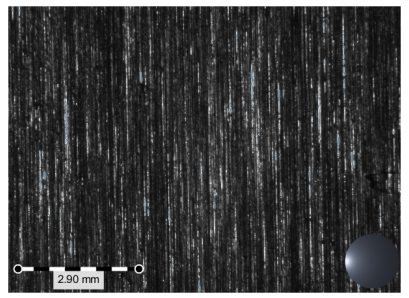

(e) Metal: $r_{S}=0 \%$

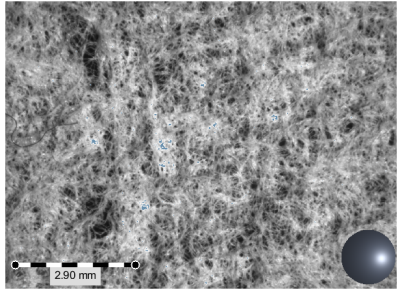

(c) Paper: $r_{S}=0 \%$

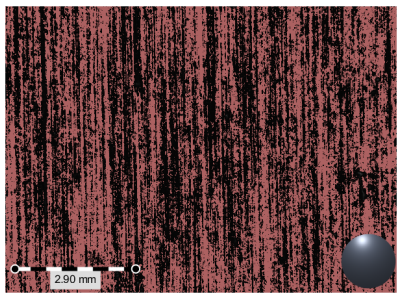

(f) Metal: $r_{S}=51 \%$

Figure 3: RTI images of a paper (a-c) and a metallic (d-f) industrial surface samples (12.4 MPix monochromatic camera - Exposure Time $\left.\left(E_{t}\right)=60 \mathrm{~ms}\right)$

The percentage of non-measured pixels, in the presented image acquisition, is named $r_{S}$. Blue pixels refer to underexposed pixels while red ones are overexposed. The first row, corresponding to the paper, have small $r_{S}$ values as it is a Lambertian surface. On the contrary, the metal images present larger $r_{S}$. It corresponds to non-measured part of the signal. Moreover, in many industrial 85 applications such as visual inspection, the critical points are often associated with high local variations of reflectance implying large $r_{S}$ values. These nonmeasured pixels make it impossible to robustly discriminate the anomalies and evaluate their criticality in terms of perception. The work presented in this article aims to answer this issue by proposing an adaptive coupling of HDR and

90 RTI imaging techniques [27]. 
The limited dynamic range of the sensor results, for RTI acquisitions, in two issues. The first one is specific to RTI and concerns the pixel-level dynamic. Indeed, varying the directions of illumination during the RTI acquisition causes for each pixel quick and high amplitude variations: low elevation angles often generate extremely low luminance values, while lighting directions associated to specular lobe generate very high luminance values. The maps presented in Figure 4 illustrate this pixel-level dynamic limitation.

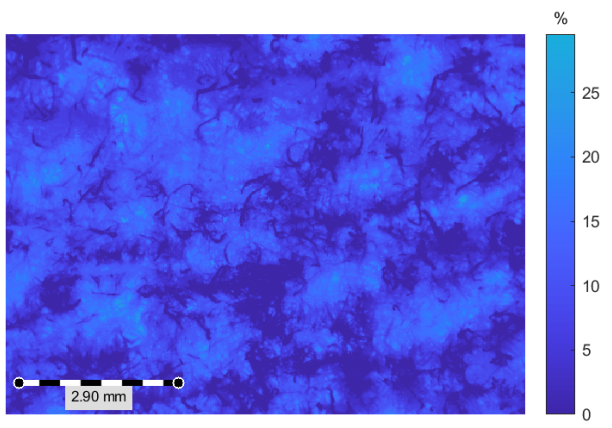

(a) Paper sample

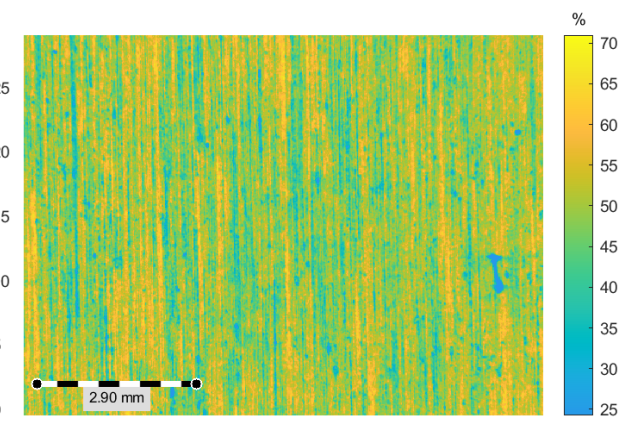

(b) Metallic brushed finished surface

Figure 4: RTI pixel level dynamic limitation: Percentages of non-measured points in RTI acquisitions with 149 homogeneous angular positions

Each point represents the percentage of RTI acquisition angles for which the luminance could not be estimated (undersaturated and oversaturated value). It is observed that even if the material is globally diffuse, certain points have not been measured for up to $30 \%$ of the RTI acquisition positions (Figure $4 \mathrm{a}$ ). This is due, in this case, to the topography of the surface, which has a heterogeneous material density. For the brushed metallic sample (Figure 4p), it is observed that up to $70 \%$ of RTI acquisition directions produce saturated values for certain pixels. This configuration is frequent for glossy surfaces, and/or if the surface presents an oriented texture (anisotropic). Indeed, for such configuration the amount of light reflected when the direction of illumination is orthogonal to the direction of texturing is often very high, and inversely.

The second issue concerns the image-level dynamic as within the same RTI acquisition image the difference in the response between two pixels can be very high. This may vary with the angle of incidence of the light, but this quantity is directly related to the intrinsic reflectance of the inspected surfaces. In the case of surfaces with local specularities or for composite surfaces, the dynamics of the reflectance response can largely exceed the range of the dynamic of the sensor,

115 which leads to a significant loss of information (sensor saturation), as illustrated in Figure 5, which represents, for each RTI acquisition angle, the percentage of non-measured points in the obtained reflectance image. It is observed that the adjustment of the exposure time, which is manually tuned by the operator for a conventional RTI acquisition, does not make it possible to capture images correctly exposed for all the acquisition directions. This is particularly the 
case for the brushed surface, where a large percentage of saturated values is obtained for the directions of illumination which are close to being orthogonal to the direction of the surface texture.

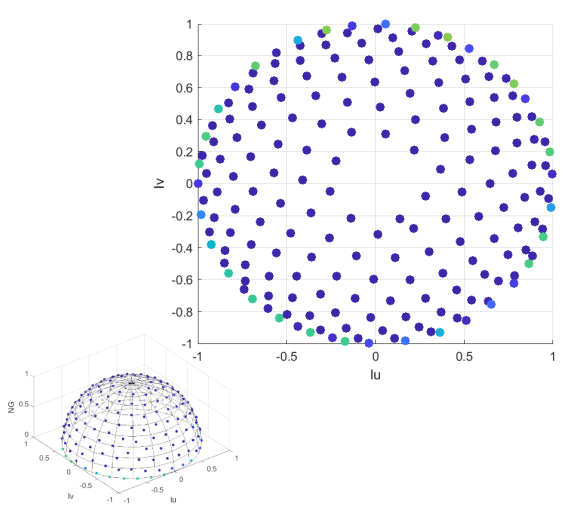

(a) Paper sample

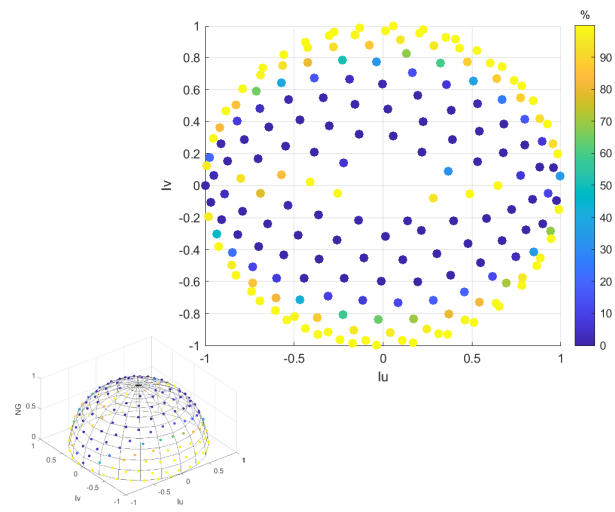

(b) Metallic brushed finished surface

Figure 5: RTI image-level dynamic limitation: Percentages of non-measured points for all illumination directions

These two limitations can also alter the quality of the RTI derived feature maps (see flowchart presented in Figure 1). For instance, RTI stereophotometric acquisitions allow to estimate the normal field of the surface, and thus to determine the slopes and curvatures [23. As example, two slope maps obtained from RTI acquisitions on the same surface at different exposure times $\left(E_{t}\right)$ are presented in Figure 6. It is observed that the arbitrary tuning of $E_{t}$ has a significant influence on the estimation of the normal field, which could possibly be detrimental for subsequent analyses.

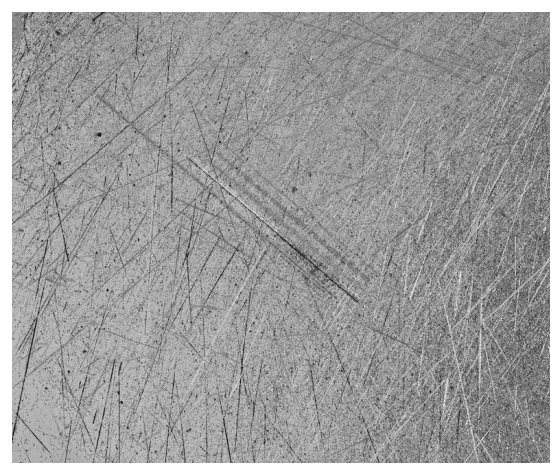

(a) $D_{x}, E_{t}=125 \mathrm{~ms}$

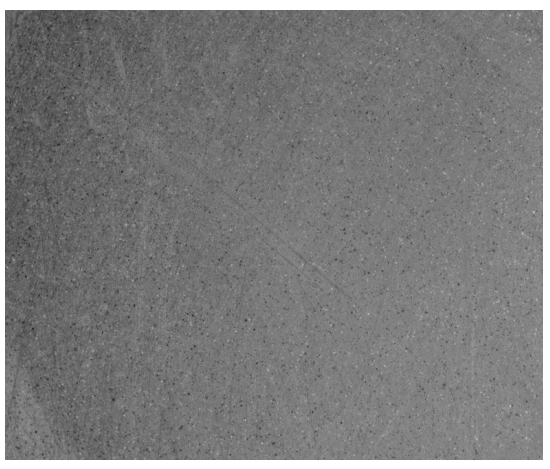

(b) $D_{x}, E_{t}=500 \mathrm{~ms}$

Figure 6: RTI-derived $D_{x}$ slopes maps obtained from acquisitions of a metallic sample captured at different exposure times $\left(E_{t}\right)$ 


\section{Proposed method for High Dynamic RTI acquisitions}

The proposed method aims at addressing these two dynamic limitations. Moreover, our implementation of High Dynamic RTI (HD-RTI) is fully automatic and adaptive: the number of required shots for each illumination direction as well as their corresponding exposure times are automatically set according to the response of the observed surface. The general flowchart of the proposed HD-RTI methodology is presented in Figure 7. Furthermore the implemented method along with its algorithm are detailed below.

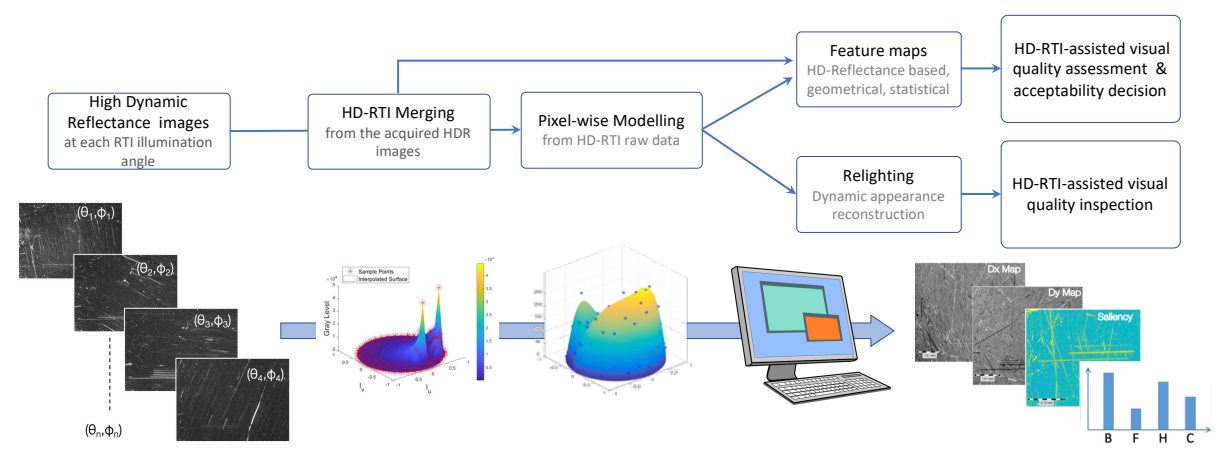

Figure 7: General flowchart of the implementation of HD-RTI

The proposed method was built on the approach of Martinez et al. 28] by adapting it to RTI acquisitions and automating the parameters tuning. The principle is to automatically adjust the number of shots (at each direction of illumination), as well as the $E_{t}$ values according to the surface response at each acquisition angle. A normalization operation is then performed in order to obtain consistent information for all acquisition illumination directions, which is essential for the RTI analysis and modelling process. The main steps of the proposed method are the estimation of the Camera Response Function (CRF), the selection of the shots and the image reconstruction. They are presented below and detailed in Algorithm 1 .

\subsection{Estimation of the CRF}

The method requires first to estimate the CRF [30, 31]. The estimation of the CRF does not have to be renewed for each HD-RTI acquisition. It is indeed possible to reuse a known CRF as long as the same camera is used. To initialize the calculation, a number of reference pixels is chosen, usually from 100 to 5000 distributed homogeneously over the image. This choice is in practice a compromise between the computing time and the noise level of the estimated CRF which tends to increase when the number of reference pixels is too low. Several images are then acquired with different $E_{t}$ to access the entire luminous response of the surface. Finally, the CRF is approximated by the minimization of the objective function $\mathcal{O}$ expressed in equation 1 . The obtained $\mathrm{CRF}$ for our 


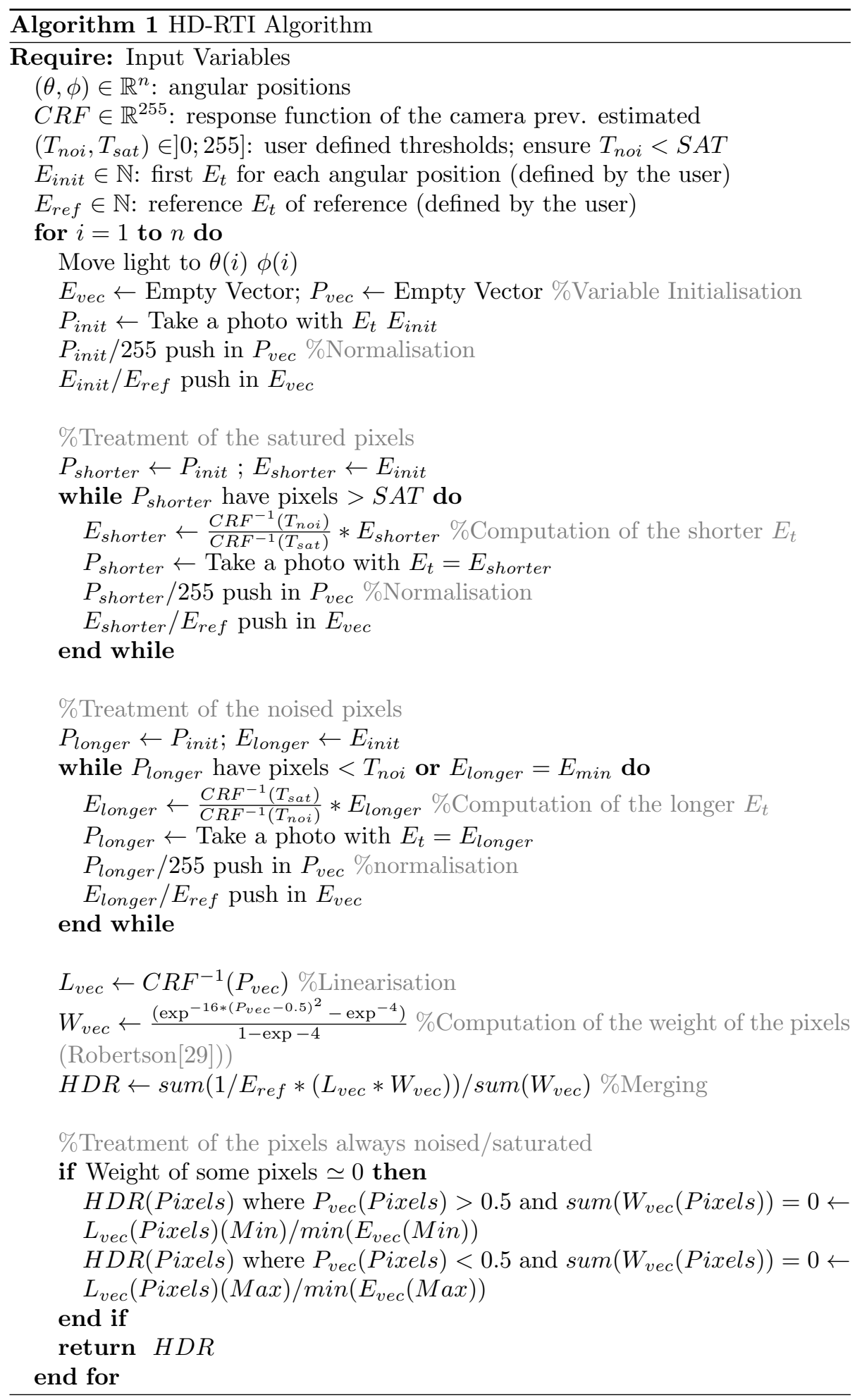


RTI setup is presented in Figure $8 \mathrm{a}$

$$
\mathcal{O}=\sum_{i=1}^{N} \sum_{j=1}^{P}\left\{\left(Z_{i j}\left[g\left(Z_{i j}\right)-\ln E_{i}-\ln \Delta t_{j}\right]\right\}^{2}+\lambda \sum_{z=Z_{\min }+1}^{Z_{\max }-1}\left[w(z) g^{\prime \prime}(z)\right]^{2}\right.
$$

Where $\left\{\begin{array}{l}g\left(Z_{i j}\right) \text { corresponds to the CRF to be determined } \\ Z_{i j} \text { is the value of the pixel i with the exposure time } \mathrm{j} \\ E_{i} \text { is the irradiance of the pixel i } \\ w \text { is a weighting function }\end{array}\right.$
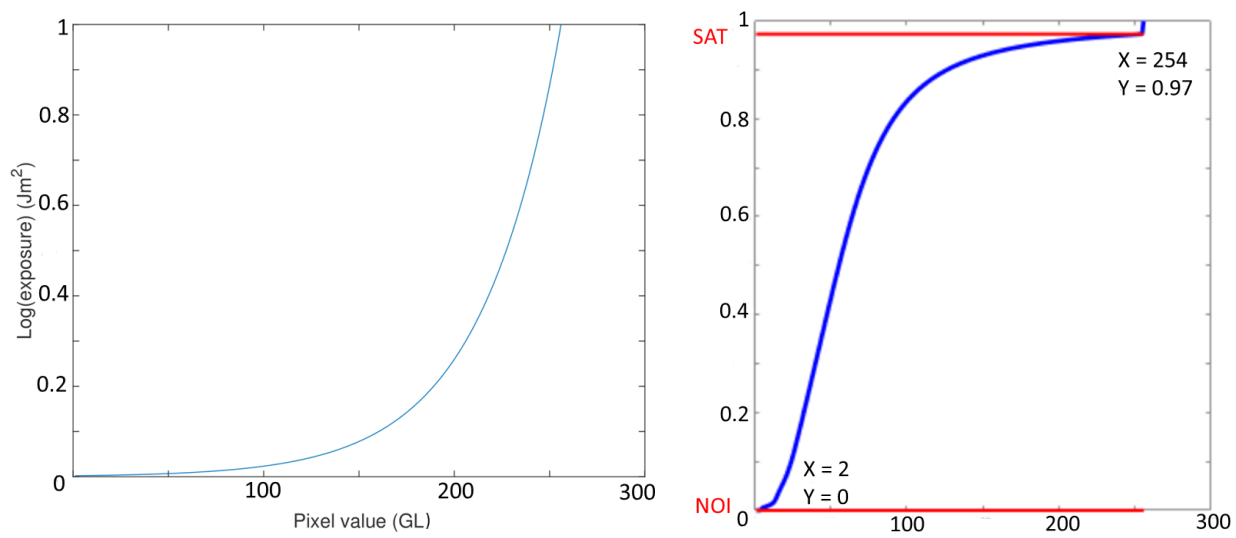

(a) Estimation of the Camera Response Function (b) Cumulative histogram of an LDR image (CRF) of our acquisition sensor (AVT MANTA G 507B)

Figure 8: Schema of a CRF and a cumulative histogram

\subsection{Number of shots and associated $E_{t}$}

We propose a method that automatically adapts for each light direction, in an RTI acquisition, the number of shots as well as their associated $E_{t}$ values. The method is based on the Adaptive Exposure Estimation (AEE) technique [28, adapted to the case of RTI acquisitions. Three pre-set parameters are used: noise $\left(T_{n o i}\right)$, saturation level $\left(T_{s a t}\right)$ and a threshold $\left(S_{p}\right)$ which corresponds to the percentage of saturated pixels considered acceptable. At each illumination direction, a first image is acquired with an arbitrary $E_{t}$ (user choice). Then recursively, other images are acquired with a longer and/or shorter $E_{t}$, respectively $E_{s}$ and $E_{l}$, automatically determined using eq. 2. Finally, the cumulative histogram of the image (Figure $8 \mathrm{~b}$ ) allows to determine whether it has a selected proportion of underexposed or overexposed pixels. If a threshold is exceeded, 
other shot(s) are carried out with longer and/or shorter $E_{t}$.

$$
\left\{\begin{array}{c}
E_{s}=E_{t} * \frac{C R F^{-1}\left(T_{\text {noi }}\right)}{C R F^{-1}\left(T_{\text {sat }}\right)} \\
E_{l}=E_{t} * \frac{C R F^{-1}\left(T_{\text {sat }}\right)}{C R F^{-1}\left(T_{\text {noi }}\right)}
\end{array}\right.
$$

The algorithm stops when the ratio of correctly exposed (unsaturated) pixels in the cumulative histogram of the image is satisfactory, otherwise all available $E_{t}$ are used. It is also possible to define (by the user) a maximum or minimum number of images to acquire if necessary. The parameters $T_{\text {noi }}$ and $T_{\text {sat }}$ influence the SNR (Signal-To-Noise ratio, estimated pixel-wise equation 33: the closer the $T_{n o i}$ and $T_{\text {sat }}$ levels are, the higher the mean SNR of the resulting HDR data. However, more shots increases both acquisition time and data volume.

$$
S N R_{x y}=20 \times \log _{10}\left(\frac{\bar{E}_{x y}}{\sigma_{x y}}\right)
$$

For each pixel $(x y)$ of the acquired image, $\bar{E}_{x y}$ and $\sigma_{x y}$ correspond to the mean and the standard deviation of the obtained HDR values respectively.

\subsection{HD-RTI images reconstruction}

This step consists in merging the LDR frames acquired to generate HDR stereo-images for each acquisition direction. It is then also possible to extract (for each pixel) the set of high dynamic luminance values and post-process these data to meet the needs of the visual quality inspection (modelling, relighting, dedirection of the HDR reflectance information requires three main steps $S_{i=1 \ldots 3}$, which are the linearization, weighting and LDR data merging [29, 30, 32.

(S1 - Linearisation). This operation consists in correcting the non-linearity of the camera. The response of the camera generally has a linear response only in the middle of the $C R F$ (see Figure 8a) while at its borders it exhibits a logarithmic / exponential behaviour. The linearization is carried out from the inverse of the $C R F$, as described in equation 4

$$
P_{\text {lin }}=C R F^{-1}(P)
$$

Where $\mathrm{P}$ is the pixel value.

(S2 - Weighting). - In this step, a function enabling to weight the pixels of 165 an LDR image acquisition according to their values, i.e., their distance to the fixed noise $\left(T_{n o i}\right)$ and saturation $\left(T_{\text {sat }}\right)$ thresholds, is saught. This weighting is intended to attenuate potential artefacts that may cause blooming or noise on the reconstructed HDR image. The most often used weight functions are Box, Robertson, Hat or Deb97 (Debevec 1997) [30. The weight functions are illustrated in the Figure 9 


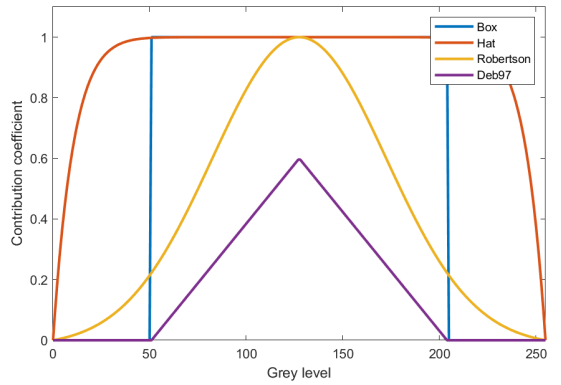

Figure 9: Weight functions

(S3 - Merging). - This operation is carried out independently for each light direction and consists in merging the LDR images acquired with different $E_{t}$, taking into account the $T_{n o i}$ and $T_{\text {sat }}$ fixed by the user, the selected weight function and the CRF. For each pixel, the HDR luminance value is obtained by 175 averaging the values extracted from each LDR image weighted by the CRF and the weight function. The high dynamic luminance values obtained at each pixel from the RTI acquisition directions must be consistent with each other, and not associated with a relative image level reflectance value as for conventional HDR imaging. To answer this issue, a reference exposure time $E_{t}^{r e f}$ is empirically chosen allowing then to calculate all HDR luminance relative to this reference, by implementing for each LDR acquisition frame $i$ the relative ratio $R_{E}=E_{t}^{i} / E_{t}^{r e f}$. The only condition to be respected in order to compare the HDR data with others is to use the same $E_{t}^{r e f}$ during their acquisitions. Indeed, the HDR data will be equivalent to scene with an exposure time of $E_{t}^{r e f}$ without the limitation of the sensor.

\section{Evaluation and results}

\subsection{RTI setup}

The acquisitions were carried out with a custom device (Figure 10a) allowing the implementation of the HD-RTI method. The image sensor is a monochromatic $2 / 3$ active pixel-type CMOS sensor, with a resolution of 12.4 MPix $(4112 \times 3008)$. The camera is equipped with a precision micro-imaging modular lens system with a motorized magnification and focus. This vision system makes it possible to adapt the field of view and to quickly focus on the area of interest. Regarding the light source, we use a white uniform light (high power 195 LED) equipped with a lens to limit the solid angle of the light to 15 degrees. In order to carry out RTI acquisitions, the light source is mounted on a motorized hoop of radius $35 \mathrm{~cm}$ capable of positioning the source in any position in the hemisphere of the same radius around the object by varying ( $\phi$ and $\theta)$ angles. All the parameters of the HD-RTI acquisition process can be controlled with a user interface (Figure 10b), which also allows the visualization of acquired data and post-processed features mappings. 


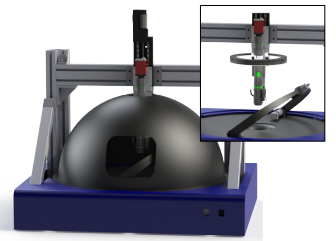

(a) Acquisition device

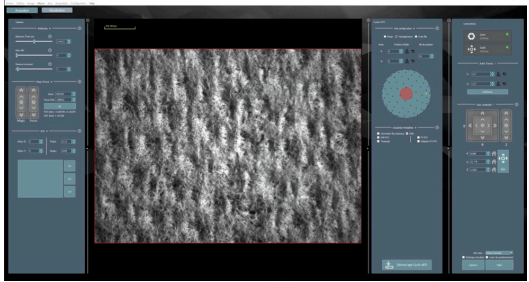

(b) User Interface

Figure 10: Custom acquisition system for HD-RTI

The method is applied on 4 samples with distinct properties in terms of isotropy and reflectance (Figure 11). The first surface sample (Surf-1) is an industrial matte paper sample. Its roughness is isotropic. Surf-2 is a gray fluoropolymer calibration patch with a high diffuse reflectance that reflect $40 \%$ of the light. It exhibits highly Lambertian behavior and can be used for light calibration in photography. Surf-3 is a brushed metal sample (hot rolling). Surf-4 is a microblasted on rough planed silver sample. The acquisitions on these four samples were carried out with a field of view of $6.89 \times 5.11 \mathrm{~mm}$ (pixel 210 size of approx. $1.7 \mathrm{\mu m}^{2}$, with 192 lighting angles distributed homogeneously (see Figure 5), at 20 different areas for each one.

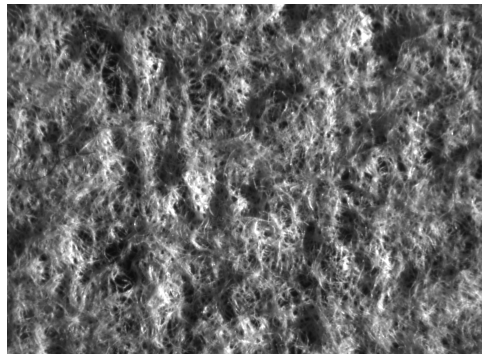

(a) Surf-1 : Paper

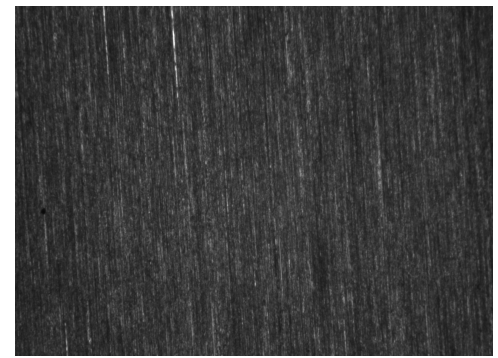

(c) Surf-3 : Metal

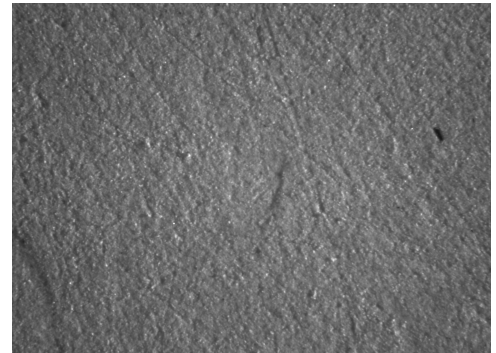

(b) Surf-2 : Polymer

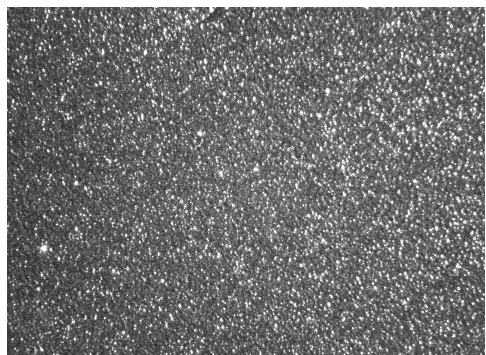

(d) Surf-4 : Silver

Figure 11: Images of Surf-[1-4] composing the dataset 


\subsection{Overall assessment of the HD-RTI method}

The first overall evaluation of the method on the four surface samples, presented above, consists in comparing RTI (noted LDR raw) and HD-RTI obtained data on Surf-[1-4]. As expected, the more a surface has a high response am215 plitude, the larger the number of images required is (table 1) at each lighting direction. We also observe that the standard deviation of Lambertian-kind surfaces is lower. This shows that the system is self-adaptive both as a function of the type of surface observed and of its response amplitude at the different acquisition angles. The comparative analysis of dynamic ratios (table 2 ) also shows

\begin{tabular}{l|c|c|c|c} 
& Surf-1 & Surf-2 & Surf-3 & Surf-4 \\
\hline Total number of light directions. & 192 & 192 & 192 & 192 \\
\hline Number of shots (LDR) $\mu(\sigma)$ & $386(0)$ & $347.2(26.6)$ & $397.8(3.6)$ & $486(6.3)$ \\
\hline Max. nb of images by direction $\mu(\sigma)$ & $2(0)$ & $2.2(0.4)$ & $3(0)$ & $3(0)$ \\
\hline Min. nb of images by direction $\mu(\sigma)$ & $2(0)$ & $1.2(0.4)$ & $2(0)$ & $2(0)$ \\
\hline Number of images by direction $\mu(\sigma)$ & $2(0)$ & $1.8(0.4)$ & $2.1(0.2)$ & $2.5(0.5)$
\end{tabular}

Table 1: Mean $(\mu)$ and standard deviation $(\sigma)$ of HD-RTI acquisitions indicators on surface samples Surf-[1-4]

\begin{tabular}{|c|c|c|c|c|c|c|c|c|c|c|c|c|}
\hline \multirow{3}{*}{$\begin{array}{c}\text { Mean } \mu(\sigma) \\
\text { Pixel-levels } \\
\text { dynamic ratio }\end{array}$} & \multicolumn{3}{|c|}{ Surf-1 } & \multicolumn{3}{|c|}{ Surf-2 } & \multicolumn{3}{|c|}{ Surf-3 } & \multicolumn{3}{|c|}{ Surf-4 } \\
\hline & \multirow{2}{*}{\begin{tabular}{|l|} 
LDR raw \\
$102 \quad$ (39) \\
\end{tabular}} & \multicolumn{2}{|c|}{ HD-RTI } & \multirow{2}{*}{\begin{tabular}{|l|} 
LDR raw \\
$113 \quad(38)$ \\
\end{tabular}} & \multicolumn{2}{|c|}{ HD-RTI } & \multirow{2}{*}{\begin{tabular}{|l|} 
LDR raw \\
255 (6)
\end{tabular}} & \multicolumn{2}{|c|}{ HD-RTI } & \begin{tabular}{|l|} 
LDR raw \\
\end{tabular} & \multicolumn{2}{|c|}{ HD-RTI } \\
\hline & & 58 & (15) & & 61 & (8) & & 1336 & (456) & 109 (33) & 454 & (184) \\
\hline $\begin{array}{l}\text { Image-levels } \\
\text { dynamic ratio }\end{array}$ & 115 (27) & 55 & (10) & 125 (24) & 57 & (4) & $255(0)$ & 1130 & (216) & \begin{tabular}{|ll}
99 & (27)
\end{tabular} & 395 & (127) \\
\hline
\end{tabular}

\begin{tabular}{|c|ccc|cc|cc|c|c|c|c|c|c|c|c|c|c|}
\hline \multirow{2}{*}{ Min $\mu(\sigma)$} & \multicolumn{4}{|c|}{ Surf-1 } & \multicolumn{4}{|c|}{ Surf-2 } & \multicolumn{4}{c|}{ Surf-3 } & \multicolumn{4}{c|}{ Surf-4 } \\
\cline { 2 - 16 } & LDR raw & HD-RTI & LDR raw & HD-RTI & LDR raw & HD-RTI & LDR raw & \multicolumn{1}{|c|}{ HD-RTI } \\
\hline $\begin{array}{c}\text { Pixel-levels } \\
\text { dynamic ratio }\end{array}$ & 6 & $(2)$ & 6 & $(2)$ & 8 & $(2)$ & 9 & $(2)$ & 12 & $(6)$ & 142 & $(80)$ & 7 & $(2)$ & 28 & $(14)$ \\
\hline $\begin{array}{c}\text { Image-levels } \\
\text { dynamic ratio }\end{array}$ & 67 & $(39)$ & 32 & $(11)$ & 76 & $(24)$ & 49 & $(8)$ & 255 & $(0)$ & 744 & $(183)$ & 45 & $(12)$ & 199 & $(66)$ \\
\hline
\end{tabular}

\begin{tabular}{|c|c|c|c|c|c|c|c|c|c|}
\hline \multirow{2}{*}{$\operatorname{Max} \mu(\sigma)$} & \multicolumn{2}{|c|}{ Surf-1 } & \multicolumn{2}{|c|}{ Surf-2 } & \multicolumn{3}{|c|}{ Surf-3 } & \multicolumn{2}{|c|}{ Surf-4 } \\
\hline & \begin{tabular}{|l} 
LDR raw \\
\end{tabular} & HD-RTI & LDR raw & HD-RTI & LDR raw & & -RTI & LDR raw & HD-RTI \\
\hline $\begin{array}{c}\text { Pixel-levels } \\
\text { dynamic ratio }\end{array}$ & 255 (0) & 301 (81) & 255 (0) & 740 (869) & 255 (0) & 23823 & (7682) & 255 (0) & 5018 (1165) \\
\hline $\begin{array}{l}\text { Image-levels } \\
\text { dynamic ratio }\end{array}$ & 156 (74) & \begin{tabular}{|ll}
78 & $(14)$
\end{tabular} & 199 (65) & 69 (11) & $255(0)$ & 1705 & (438) & 167 (63) & $844 \quad$ (313) \\
\hline
\end{tabular}

Table 2: RTI vs HD-RTI dynamic ratio indicators on Surf-[1-4]

220 higher values for HD-RTI. It confirms that the information was only partially measured with the conventional RTI method. Moreover, it is also observed that the mean ratios are increasing in the case of the HD-RTI acquisitions. It corresponds to the expected reflectance behaviour of the studied surfaces, whereas 
this up-trending evolution is not clear in the RTI data. Lastly, the large values of pixel-level ratio recorded also confirms the necessity and relevance of the proposed approach for enhanced RTI. These results are corroborated by the distribution of the image-level and pixel-level dynamic ratios. The distributions obtained for Polymer are presented in Figure 12 (similar results are obtained for the three other samples).

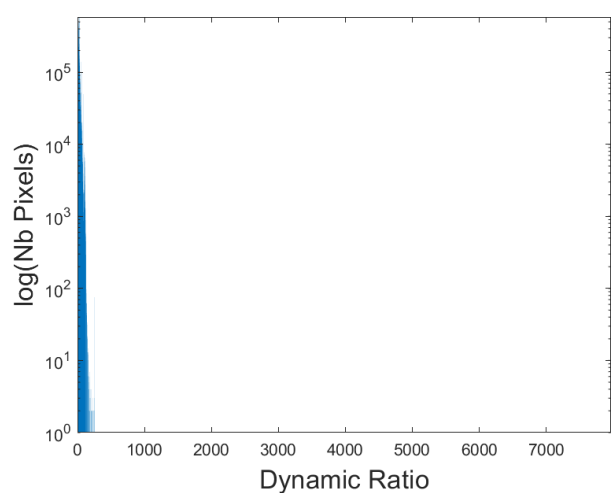

(a) RTI

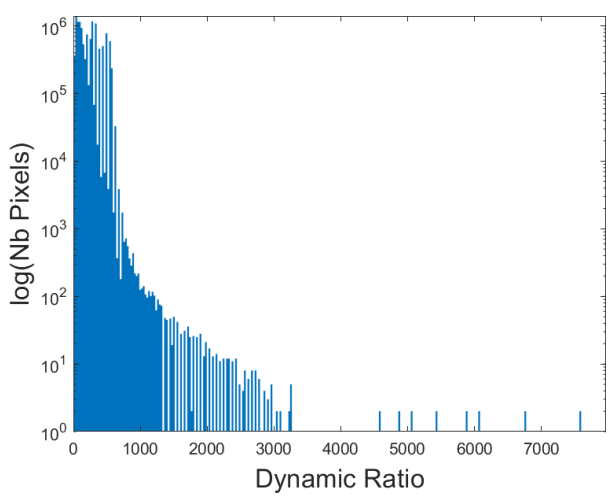

(b) HD-RTI

Figure 12: Polymer pixel-level dynamic ratio distribution RTI acquisition, and inevitably lead to a large number of non-measured (over or underexposed) points. These results are confirmed by the comparison of the $3 \mathrm{D}$ reflectance maps presented in Figure 14 


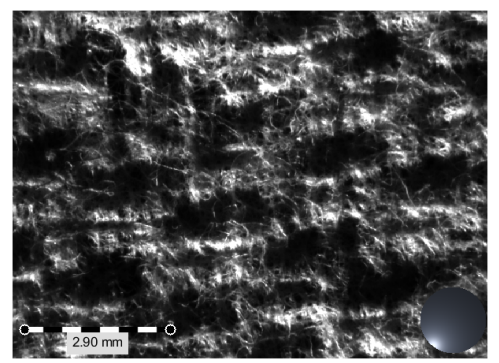

(a) Surf-1 - LDR raw RTI acquisition; $\left(\theta=232^{\circ}, \phi=8^{\circ}\right)$

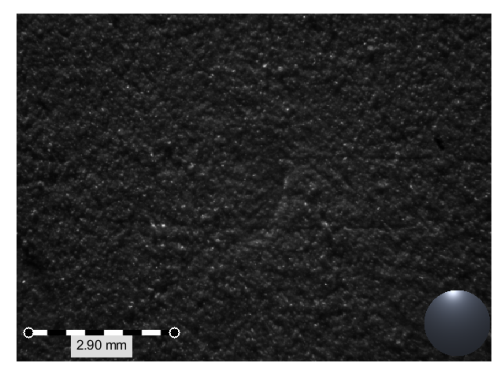

(c) Surf-2 - LDR raw RTI acquisition; $\left(\theta=97^{\circ}, \phi=5^{\circ}\right)$

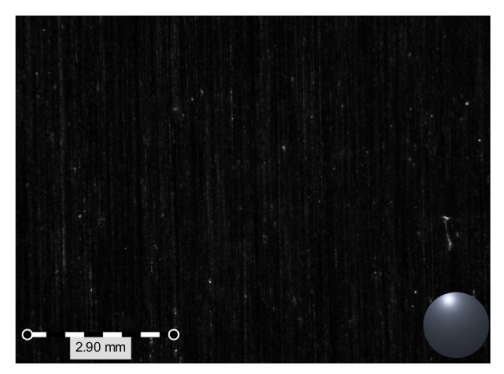

(e) Surf-3 - LDR raw RTI acquisition; $\left(\theta=106^{\circ}, \phi=35^{\circ}\right)$

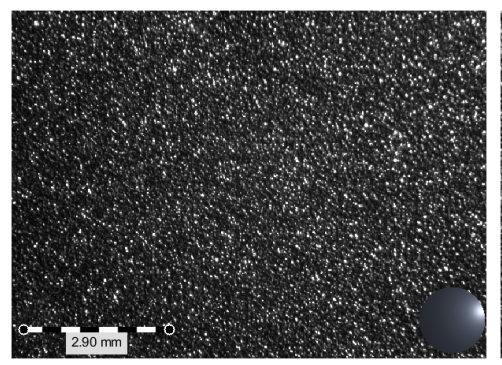

(g) Surf-4 - LDR raw RTI acquisition; $\left(\theta=17^{\circ}, \phi=20^{\circ}\right)$

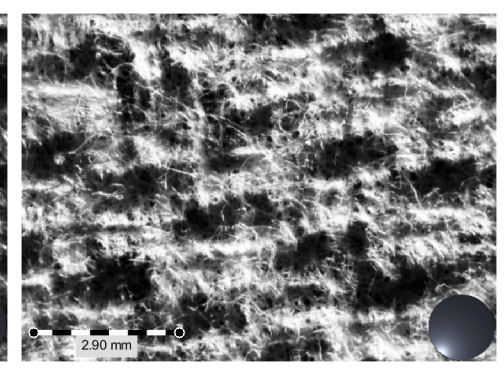

(b) Surf-1 - HD-RTI tone mapped; $\left(\theta=232^{\circ}, \phi=8^{\circ}\right)$

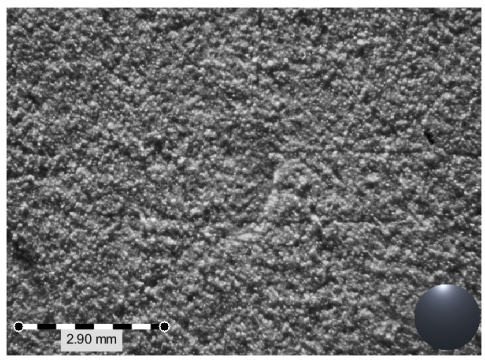

(d) Surf-2 - HD-RTI tone mapped; $\left(\theta=97^{\circ}, \phi=5^{\circ}\right)$

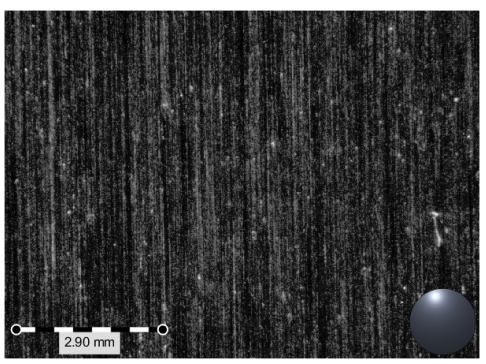

(f) Surf-3 - HD-RTI tone mapped; $\left(\theta=106^{\circ}, \phi=35^{\circ}\right)$

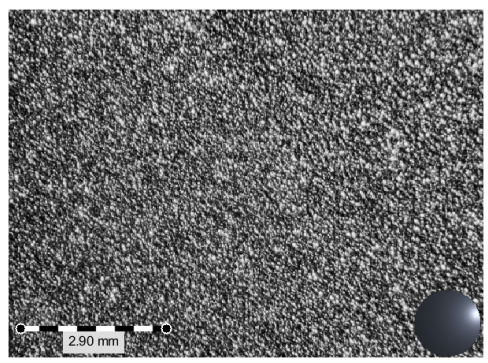

(h) Surf-4 - HD-RTI tone mapped; $\left(\theta=17^{\circ}, \phi=20^{\circ}\right)$

Figure 13: Comparison of the LDR raw acquisition and the tone map of the HDR data for the light direction $(\theta, \phi)$, on Surf-[1-4] 


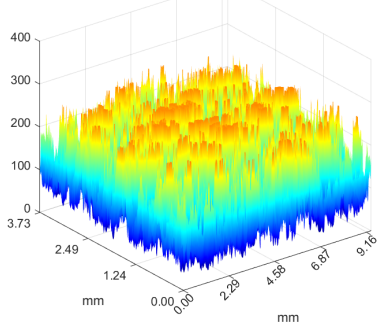

(a) Surf-1 - LDR raw reflectance surface $\left(\theta=106^{\circ}, \phi=35^{\circ}\right)$

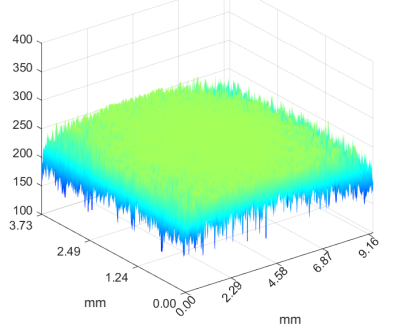

(c) Surf-2 - LDR raw reflectance surface $\left(\theta=134^{\circ}, \phi=41^{\circ}\right)$

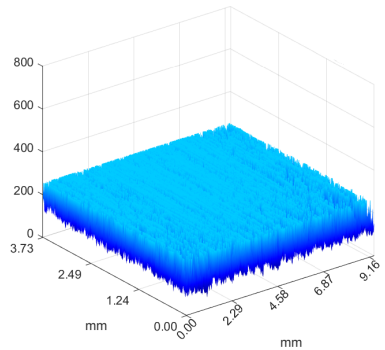

(e) Surf-3 - LDR raw reflectance surface $\left(\theta=192^{\circ}, \phi=56^{\circ}\right)$

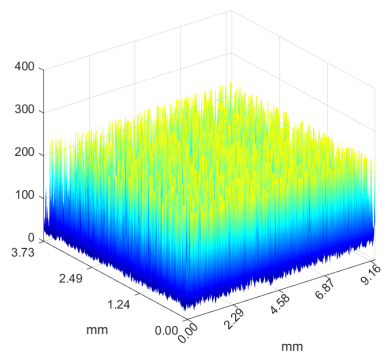

(g) Surf-4 - LDR raw reflectance surface $\left(\theta=17^{\circ}, \phi=20^{\circ}\right)$

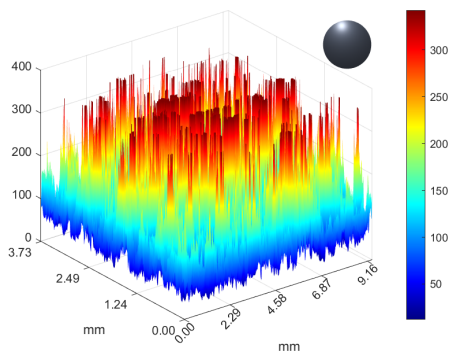

(b) Surf-1 - HD-RTI reflectance surface $\left(\theta=106^{\circ}, \phi=35^{\circ}\right)$

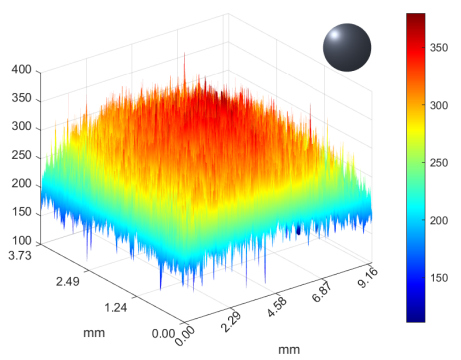

(d) Surf-2 -HD-RTI reflectance surfaces $\left(\theta=134^{\circ}, \phi=41^{\circ}\right)$

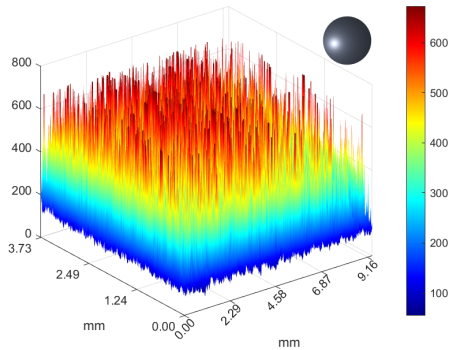

(f) Surf-3 - HD-RTI reflectance surface $\left(\theta=192^{\circ}, \phi=56^{\circ}\right)$

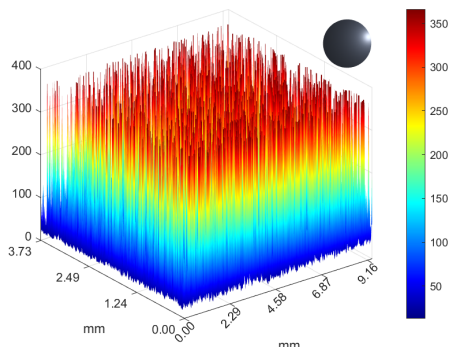

(h) Surf-4 -HD-RTI reflectance surfaces $\left(\theta=17^{\circ}, \phi=20^{\circ}\right)$

Figure 14: Comparison of the angular reflectance values, extracted for the light direction $(\theta, \phi)$, on Surf-[1-4] 

results are presented in Figure 15. Due to the saturation of the RTI 99 when the

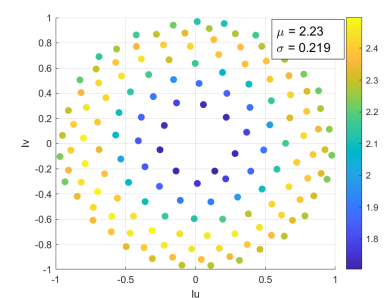

(a) $\frac{\mathrm{RTI}_{99 \%}}{\mathrm{RTI}_{40 \%}}$

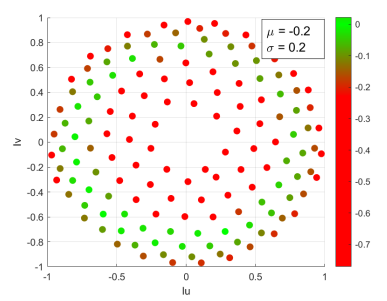

(c) $\frac{\mathrm{RTI}_{99 \%}}{\mathrm{RTI}_{40 \%}}-\frac{99}{40}$

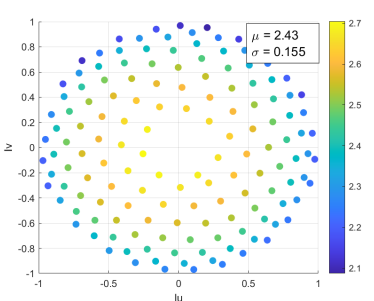

(b) $\frac{\text { HD-RTI }_{99 \%}}{\text { HD-RTI }_{40 \%}}$

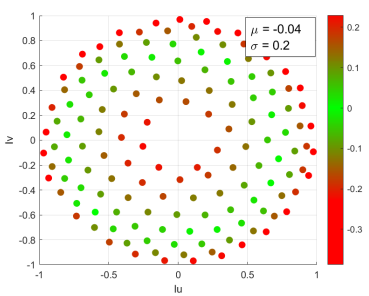

(d) $\frac{\mathrm{HD}^{-R^{2}} I_{99 \%}}{\mathrm{HD}_{\mathrm{RTI}}}-\frac{99}{40}$

Figure 15: Luminance coefficients from RTI and HD-RTI acquisitions of fuoropolymer surfaces with a reflectance of $99 \%$ and $40 \%(99 / 40=2.475)$

$\mathrm{RTI}_{40}$ do not saturate, the coefficients decrease, while, the HD-RTI $99 \%$ do not saturate therefore the luminance coefficient do not decrease with high elevation. The mean of the luminance coefficients of the HD-RTI acquisitions is closer to the theorical value (2.475) than the RTI acquisitions. Moreover, the difference of coefficient between the HD-RTI acquisitions and the theoretical value can be explained by the missing values for an elevation greater than $75^{\circ}$ due to setup limitation. With HD-RTI method we can acquire the physical response of the surfaces, be closer to the visual human perception than with classical RTI acquisition. Data closer to human perception would lead to more robust analysis 
and visual assessment.

\subsection{Relighting with HD-RTI}

Reconstructed HD-RTI data can be used for relighting by virtually varying the amount of the light as well as its spatial position as a function of $\left(f_{R}\left(\theta, \phi, E_{t}\right)\right)$. This additional degree of freedom for reconstruction allows the lighting configuration to be fully adapted to the area one wishes to inspect or analyse, and is especially useful for surfaces heterogeneous in color and texture, and composite (multi-material) surfaces. To achieve this dynamic relighting, it is first necessary to build an experimental model from the HDR acquired discrete data at each acquisition angle. Then the model allow to continuously reconstruct the HDR image of the surface as a function of the lighting angle $(\phi, \theta)$. The second step then consists in converting the reconstructed HDR image into an LDR image associated with the chosen reconstruction time $\left(E_{t}\right)$. This conversion operation is described in equation 5 The pipeline for HD-RTI relighting is presented in Figure 16

$$
\operatorname{LDR}\left(\theta, \phi, E_{t}\right)=\left\{\begin{array}{cc}
\text { if } \operatorname{int}\left(H D R(\theta, \phi) \times \frac{E_{t}}{E_{t}^{r e f}}\right)>255 \text { then } & 255 \\
\text { else } & \operatorname{int}\left(H D R(\theta, \phi) \times \frac{E_{t}}{E_{t}^{r e f}}\right)
\end{array}\right.
$$

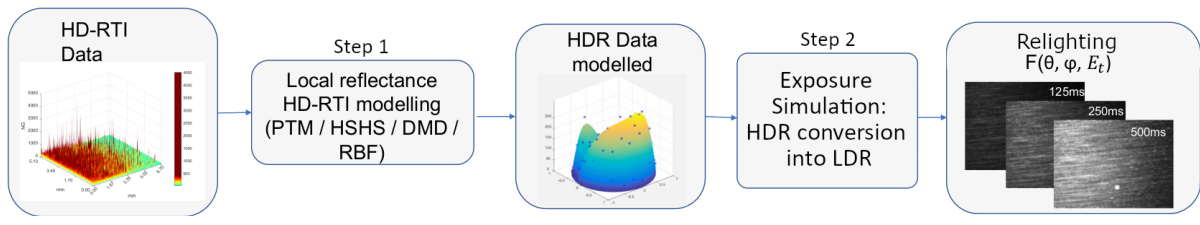

Figure 16: Pipeline of the appearance reconstruction from HDR data

The reconstruction results $\left(f_{R}\left(\theta, \phi, E_{t}\right)\right)$ are presented in table 3 and Figure 275 17. It is observed that the proposed method leads to very accurate reconstructions of the appearance (with reference to the acquired data), particularly for shaded or saturated areas. The variable $E_{t}$ allows, in addition to the conventional RTI relighting, to dynamically adapt the light exposure in relation to the amplitude of the response in the area of interest.

\begin{tabular}{|c|c|c|c|c|c|c|c|c|c|}
\hline & & & \multicolumn{2}{|c|}{ Surf-1 } & \multicolumn{2}{|c|}{ Surf-2 } & \multicolumn{2}{|c|}{ Surf-3 } & Surf-4 \\
\hline \multirow{4}{*}{$E_{t}(m s)$} & 15 & \multirow{4}{*}{$\mu(\sigma)(\%)$} & 0,8 & $(0,3)$ & 0,5 & $(0,3)$ & 3,1 & $(7,5)$ & $3,7 \quad(3,6)$ \\
\hline & 30 & & 1,4 & $(0,6)$ & 1 & $(0,6)$ & 4,4 & $(8,8)$ & $5,6 \quad(4,3)$ \\
\hline & 60 & & 3 & $(1,1)$ & 2 & $(1,4)$ & 6,5 & $(11,4)$ & $6,4 \quad(4,6)$ \\
\hline & 125 & & 4,9 & $(3,8)$ & 3,3 & $(5,4)$ & 9,5 & $(12,5)$ & $7,7 \quad(6,1)$ \\
\hline
\end{tabular}

Table 3: Mean absolute error between acquired LDR raw data (ground truth) and reconstructed images at differents $E_{t}$ from HD-RTI acquisitions (in grey-levels) 


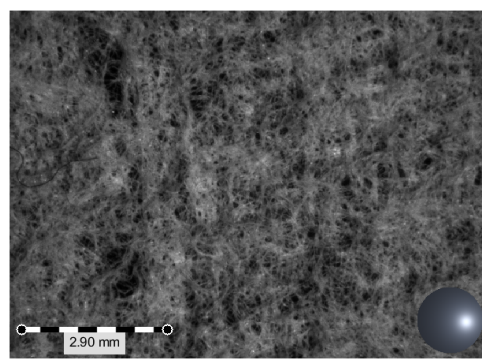

(a) Surf-1; LDR raw acquisition; $\left(\theta=353^{\circ}, \phi=60^{\circ}, E_{t}=15 \mathrm{~ms}\right)$

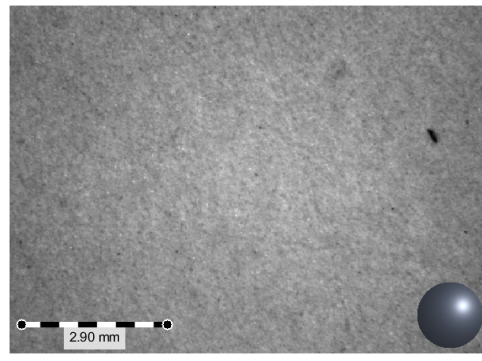

(c) Surf-2; LDR raw acquisition; $\left(\theta=36^{\circ}, \phi=61^{\circ}, E_{t}=60 \mathrm{~ms}\right)$

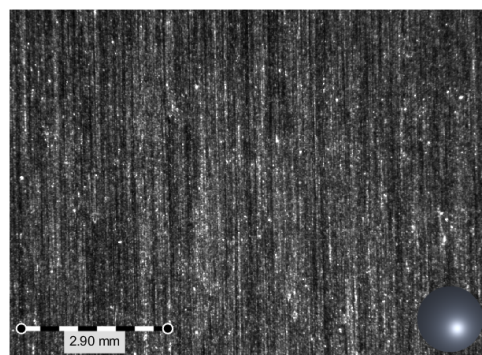

(e) Surf-3; LDR raw acquisition; $\left(\theta=307^{\circ}, \phi=69^{\circ}, E_{t}=125 \mathrm{~ms}\right)$

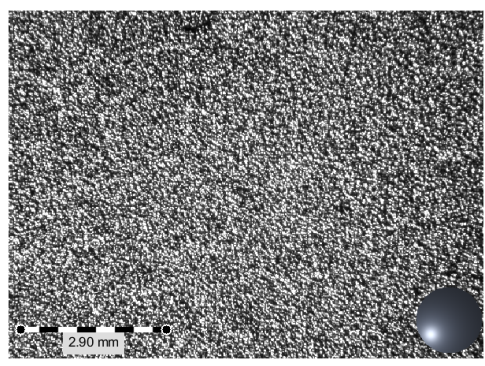

(g) Surf-4; LDR raw acquisition $\left(\theta=217^{\circ}, \phi=44^{\circ}, E_{t}=30 \mathrm{~ms}\right)$

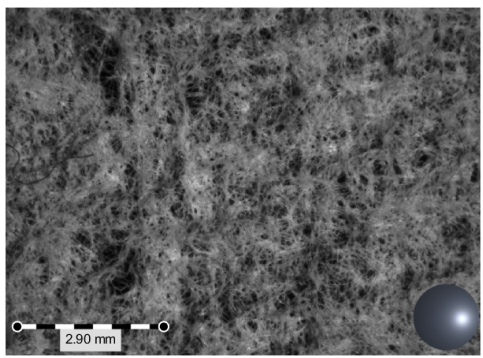

(b) Surf-1; HD-RTI relighted image; $\left(\theta=353^{\circ}, \phi=60^{\circ}, E_{t}=15 \mathrm{~ms}\right)$

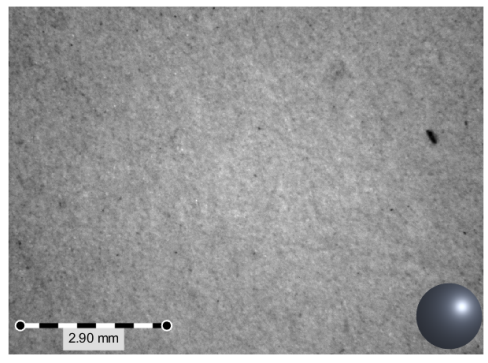

(d) Surf-2; HD-RTI relighted image; $\left(\theta=36^{\circ}, \phi=61^{\circ}, E_{t}=60 \mathrm{~ms}\right)$

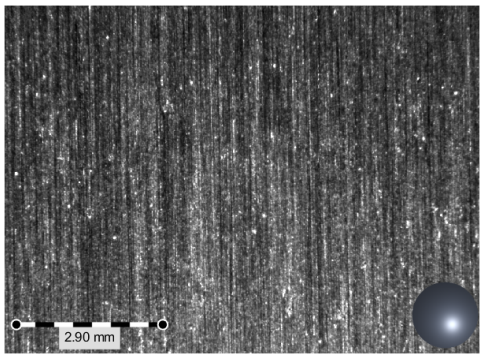

(f) Surf-3; HD-RTI relighted image; $\left(\theta=307^{\circ}, \phi=69^{\circ}, E_{t}=125 \mathrm{~ms}\right)$

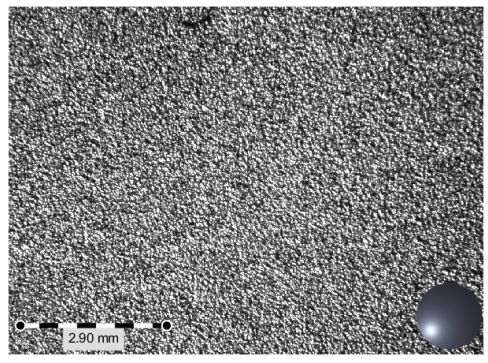

(h) Surf-4; HD-RTI relighted image; $\left(\theta=217^{\circ}, \phi=44^{\circ}, E_{t}=30 \mathrm{~ms}\right)$

Figure 17: Comparison between (left) LDR raw acquisitions (ground truth) and (right) Relighted images with DMD method, from HD-RTI acquisition. 


\subsection{Saliency map for the inspection of visual defects}

As shown in the previous section, the implementation of HD-RTI method leads to different data structure and dynamic, which improves data quality and relighting but also impact the calculation of the RTI derived feature maps. We

285 focus here on the application case of the construction of an RTI-based saliency map [33. Our purpose is to assist the inspection of visual defects. In this case, we expect the saliency map to highlight the defects.

\subsection{Experimental protocol}

The chosen surface sample is a watch dial that has been preliminarily micro290 scratched. The scratches are carried out in a controlled way so that we know their locations, directions and dimensions (Figure 18). To evaluate the difference in the results obtained, RTI and HD-RTI acquisitions were carried out on this sample, at identical resolution as Surf-[1-4] acquisitions with 149 homogeneous light positions.

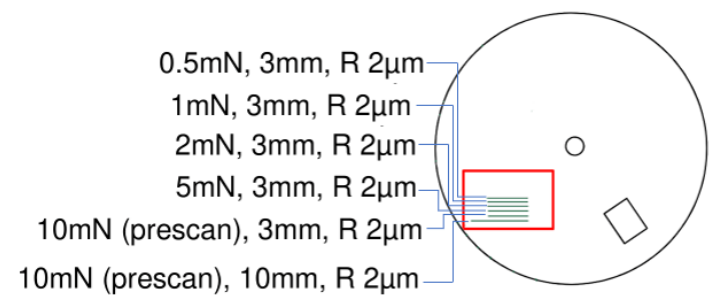

Figure 18: Representation of the dial with normalized scratches; The red rectangle is the area of the acquisitions

\subsection{Proposed approach}

The saliency is here estimated from the HD-RTI descriptors by calculating a Mahalanobis distance 34, 35. between each pixel and the mean descriptors values (equation 6). The Mahalanobis distance has the advantages to be unitless, scale invariant, and taking into account the variance of the data. This makes it particularly appropriate for this saliency estimation, based on reflectance statistical descriptors extracted from HD-RTI acquisitions. Indeed, these experimental data are by nature noisy, the descriptors can be correlated, and the quantities analyzed are of different units and scales.

$$
D_{\text {Mahal }}=\sqrt{\left(\lambda^{\prime}-\mu\right)^{T} \Sigma^{-1}\left(\lambda^{\prime}-\mu\right)},
$$

Where the matrix $\lambda^{\prime}=\left[\lambda_{1}^{\prime}, \lambda_{2}^{\prime}, \ldots, \lambda_{n}^{\prime}\right]$ is composed of the descriptors vectors of each pixel $n, \Sigma$ and $\mu=\left(\mu_{1}, \mu_{2}, \ldots, \mu_{i}\right)^{T}$ are respectively the covariance matrix and the mean descriptor vector. 
In the case of this application, the implemented descriptors are estimated from the luminance distributions of each pixel (coefficient of variation, kurtosis, skewness, entropy and energy). The saliency maps are then assessed for the two acquisitions as described previously.

\subsection{Results}

The obtained maps (Figure 19) are significantly different. A quality criterion of a saliency map is the differentiation between the background and the salient elements. In this case the background of the HD-RTI saliency map is lower, and the saliency amplitude of the anomalies (scratches and other surface defects) is higher, which means that the HD-RTI saliency assessment is more robust 310 and discriminative. This is confirmed in the associated histograms, which figure out that the mean is lower, the standard deviation higher in the HD-RTI case. These results therefore show the improvement in the quality of the processing and analyses which could be carried out from RTI acquisitions by implementing the proposed method.

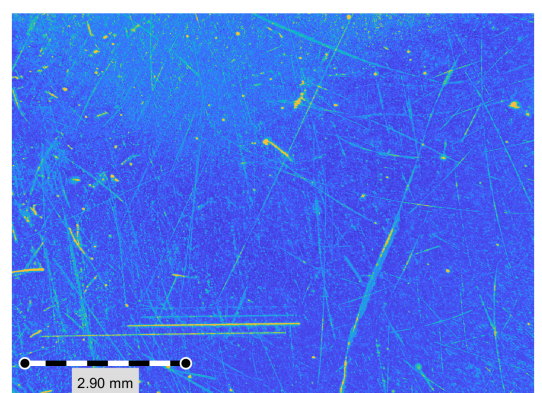

(a) RTI

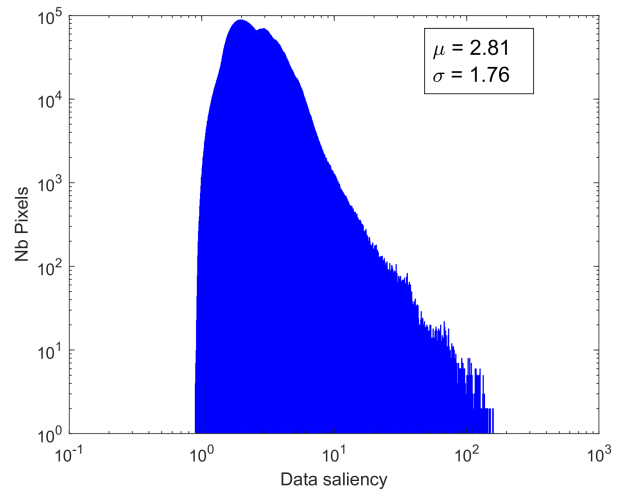

(c) RTI saliency histogram

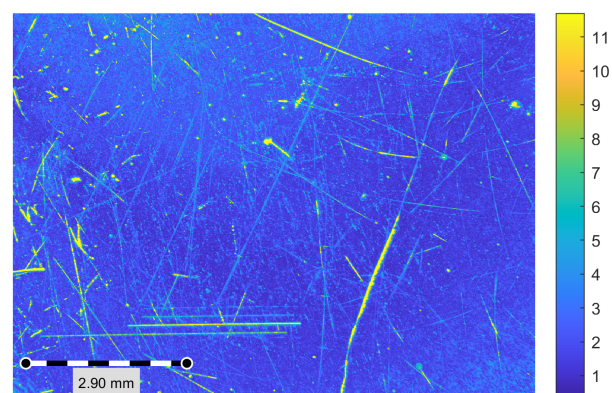

(b) HD-RTI

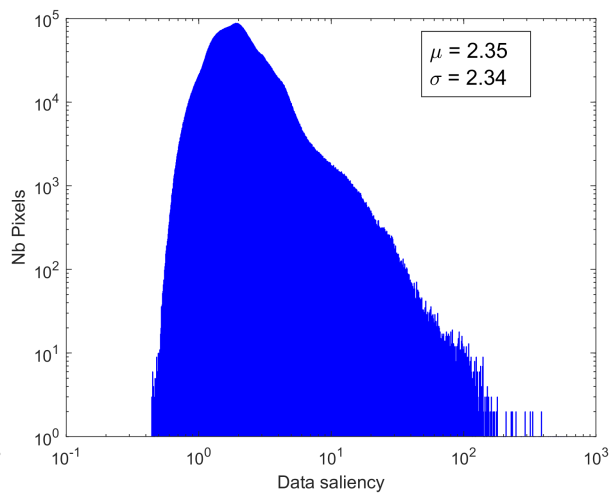

(d) HD-RTI saliency histogram

Figure 19: RTI vs HD-RTI - Saliency maps and histogram 


\section{Conclusion}

This paper presented a methodology for implementing High Dynamic Range technique for RTI acquisitions. The proposed method is self-adaptive both as a function of the type of surface observed and of its response amplitude at the different acquisition angles. The experiments were carried out on distinct surface samples in terms of roughness and reflectance enabling a non-oriented comparison of the results with RTI. The results showed that the method is particularly appropriate for heterogeneous and glossy surfaces. Indeed, these surfaces often do not allow to define an appropriate acquisition time for all the RTI acquisition angles, which inevitably induces non-measured points. In

325 terms of relighting, the proposed method leads to more accurate reconstructions particularly for shaded or saturated areas. In addition to the conventional RTI relighting, HD-RTI allows one to dynamically adapt the light exposure in relation to the amplitude of the response in the area of interest. Finally this study proved that the HD-RTI based saliency assessment is more robust and

330 discriminative, which makes it more reliable for industry applications related to quality inspection.

\section{Acknowledgments}

This work benefited of the funding of French National Research Agency (ANR) through the project NAPS (https://anr.fr/Projet-ANR-17-CE10-0005).

\section{References}

[1] Y. Castro, M. Nurit, G. Pitard, A. Zendagui, Calibration of spatial distribution of light sources in reflectance transformation imaging based on adaptive local density estimation, Journal of Electronic Imaging 29 (2020) $1-18$.

[2] T. G. Dulecha, R. Pintus, E. Gobbetti, A. Giachetti, SynthPS: a Benchmark for Evaluation of Photometric Stereo Algorithms for Cultural Heritage Applications, Proc. EG GCH, Eurographics Workshop on Graphics and Cultural Heritage, 2020.

[3] B. Martlin, C. Rando, An assessment of the reliability of cut surface characteristics to distinguish between hand-powered reciprocating saw blades in cases of experimental dismemberment, Journal of Forensic Sciences, 2020.

[4] C. Iglesias, J. Martínez, J. Taboada, Automated vision system for quality inspection of slate slabs, Computers in Industry 99 (2018) 119-129.

[5] M. Smith, R. Stamp, Automated inspection of textured ceramic tiles, Computers in Industry 43 (2000) 73-82. 
[6] H. Coules, P. Orrock, C. Seow, Reflectance transformation imaging as a tool for engineering failure analysis, Engineering Failure Analysis 105 (2019) $1006-1017$.

[7] L. Ma, Y. Liu, X. Pei, L. Shi, Turbine blade surface recovery based on photometric stereo, Vol. 11552, Proc. SPIE, Optical Metrology and Inspection for Industrial Applications VII, 2020.

[8] J. Lemesle, F. Robache, G. Le Goic, A. Mansouri, Surface reflectance: An optical method for multiscale curvature characterization of wear on ceramic-metal composites, Materials 13 (2020) 1024.

[9] G. Le Goic, Geometric quality and Appearance of surfaces: Local and global approach, Doctoral dissertation, University of Savoie Mont-Blanc, 2012 .

[10] G. Obein, J. Audenaert, G. Ged, F. Leloup, Metrological issues related to BRDF measurements around the specular direction in the particular case of glossy surfaces, Proc. SPIE 9398, Measuring, Modeling, and Reproducing Material Appearance, 2015.

[11] S. Jung, S. Woo, K. Kwon, Apparatus and method of reading texture data for texture mapping, Patent US8605101B2, 2013.

[12] T. Malzbender, D. Gelb, H. Wolters, B. Zuckerman, Enhancement of shape perception by surface reflectance transformation, HP Labs Technical reports HPL-2000-38R1 (2000) 1-3.

[13] A. Zendagui, J. Thomas, G. Le Goic, Y. Castro, Quality assessment of reconstruction and relighting from rti images: Application to manufactured surfaces, Proc. SITIS, Signal-Image Technology Internet-Based Systems (2019) 746-753.

[14] D. Ravimal, H. Kim, D. Koh, J. H. Hong, S.-K. Lee, Image-based inspection technique of a machined metal surface for an unmanned lapping process, International Journal of Precision Engineering and Manufacturing-Green Technology 7 (3) (2020) 547-557.

[15] F. Cerezci, S. Kazan, M. A. Oz, C. Oz, T. Tasci, S. Hizal, C. Altay, Online metallic surface defect detection using deep learning, Emerging Materials Research 9 (4) (2020) 1-8.

[16] G. Pitard, G. Le Goic, H. Favreliere, S. Samper, Discrete Modal Decomposition for surface appearance modelling and rendering, Proc. SPIE 9525, Optical Measurement Systems for Industrial Inspection IX, 952523, 2015.

[17] R. Pintus, T. Dulecha, A. Jaspe, A. Giachetti, Objective and Subjective Evaluation of Virtual Relighting from Reflectance Transformation Imaging Data, Proc. EG GCH, Eurographics Workshop on Graphics and Cultural Heritage, 2018. 
[18] G. Pitard, G. Le Goic, A. Mansouri, H. Favreliere, Discrete modal decomposition: a new approach for the reflectance modeling and rendering of real surfaces, Machine Vision and Applications 28 (2017) 607-621.

[19] T. Malzbender, D. Gelb, H. Wolters, Polynomial texture maps, Proc. SIGGRAPH'01, Computer graphics and interactive techniques (2001) 519-528.

[20] P. Gautron, J. Krivanek, S. Pattanaik, K. Bouatouch, A novel hemispherical basis for accurate and efficient rendering, Proc. ESGR'04 Papers, Eurographics conference on Rendering Techniques (2004) 321-330.

[21] A. Giachetti, I. Ciortan, C. Daffara, R. Pintus, Multispectral RTI Analysis of Heterogeneous Artworks, Proc. EG GCH, Eurographics Workshop on Graphics and Cultural Heritage, 2017.

[22] F. Ponchio, M. Corsini, R. Scopigno, Relight: a compact and accurate rti representation for the web, Graphical Models 105 (2019) 101040.

[23] R. J. Woodham, Gradient and curvature from the photometric-stereo method, including local confidence estimation, J. Opt. Soc. Am. A 11 (1994) 3050-3068.

[24] Y. Castro, G. Pitard, G. Le Goïc, V. Brost, A new method for calibration of the spatial distribution of light positions in free-form RTI acquisitions, Proc. O3A, Optics for Arts, Architecture, and Archaeology, 2019.

[25] Y. Castro, G. Pitard, A. Zendagui, G. Le Goic, Light spatial distribution calibration based on local density estimation for reflectance transformation imaging, Proc. SPIE QCAV, Quality Control by Artificial Vision 11172 (2019) 65-73.

[26] G. Pitard, G. Le Goic, A. Mansouri, H. Favreliere, Robust anomaly detection using reflectance transformation imaging for surface quality inspection, Proc. SCIA, Scandinavian Conference on Image Analysis 10269 (2017) 550561.

[27] M. Nurit, Y. Castro, A. Zendagui, G. L. Goïc, H. Favreliere, A. Mansouri, High dynamic range reflectance transformation imaging: an adaptive multilight approach for visual surface quality assessment, Proc. SPIE QCAV, Quality Control by Artificial Vision 11172 (2019) 276-284.

[28] M. Martínez-Domingo, E. Valero, J. Hernández-Andrés, Adaptive exposure estimation for high dynamic range imaging applied to natural scenes and daylight skies, Applied Optics 54 (2015) 241-250.

[29] F. Banterle, A. Artusi, K. Debattista, A. Chalmers, Advanced high dynamic range imaging, 2nd Edition, A K Peters/CRC Press, 2017.

[30] P. Debevec, J. Malik, Recovering high dynamic range radiance maps from photographs, Proc. SIGGRAPH '97, Computer graphics and interactive techniques (1997) 369-378. 
[31] S. Nayar, T. Mitsunaga, High dynamic range imaging: Spatially varying pixel exposures, Proc. IEE CVPR, Computer Vision and Pattern Recognition 1 (2000) 472-479.

[32] M. Robertson, S. Borman, R. Stevenson, Dynamic range improvement through multiple exposures, Proc. IEEE ICIP, International Conference on Image Processing 3 (2000) 159-163.

[33] H. Anzid, G. Le Goic, A. Bekkari, A. Mansouri, Benchmarking Saliency Detection Methods on Multimodal Image Data, Image and Signal Processing, 2018.

[34] P. C. Mahalanobis, On the generalized distance in statistics, Proceedings of the National Institute of Sciences (Calcutta) 2 (1936) 49-55.

440 [35] G. Pitard, G. Le Goic, A. Mansouri, H. Favreliere, Reflectance-based surface saliency, Proc. IEEE ICIP, International Conference on Image Processing (2017) 445-449. 